\title{
Development and User Experiences of the Learn Viena Karelian Mobile Web Game
}

\author{
Triando \\ Faculty of Computer Science \\ Free University of Bozen-Bolzano \\ Bolzano, Italy \\ DamiriBurlian.Triando@stud-inf.unibz.it
}

\author{
Leena Arhippainen \\ INTERACT Research Unit \\ University of Oulu \\ Oulu, Finland \\ Leena.Arhippainen@oulu.fi
}

\begin{abstract}
Viena Karelian is a dialect of Karelian language, which is categorized as an endangered language and requires an innovative approach to increase motivation and skills of learners. Currently, there are mainly traditional ways to learn Viena Karelian dialect such as occasional gathering and language courses are conducted by a cultural community. Therefore, gamification approach was utilized to develop a mobile web game, so beginner learners could learn in a fun and effective ways, also location-independently. A mobile web game prototype was implemented by utilizing the Laravel PHP framework and Bootstrap frontend framework, which enable the game prototype accessible on mobile web browsers. The main concept of this game was constructed by using a language learning model, which was defined with levels of easy, medium, and hard, also skill sections of listening, reading, and writing. Preliminary user experience test was conducted with 12 game experts and the result shows the game prototype was experienced as Engaging, Easy to use, Useful, Approachable and Consistent.

Keywords-gamification; revitalization; serious games; user experience; viena karelian; learning; mobile web game; language; dialect; culture
\end{abstract}

\section{INTRODUCTION}

Viena Karelian is one dialect of Karelian language. Karelian is a endangered language [1], [2] and it is divided into two dialects, which are a) Karelian Proper (Viena Karelian (North), and South Karelian) and b) Olonets Karelian (Livvi-Karelian) [1], [3], [4]. Currently, approximately less than six percentage of population of Republic of Karelia can speak Karelian language and in the Republic of Karelia, the offical language is Russian, instead of Karelian [1], [3]. There exists different estimations of Karelian speakers in Finland. According to one estimation, there are about 5,000 people who speak Karelian language daily in Finland and approximately 20,000 people who have ancestral lineage in Karelian and they can understand Karelian language to some extent [5]. According to Sarhimaa, there are about 11,000 Karelian speakers in Finland who can speak Karelian well or very well [5]. The small amount of Karelian speakers in Finland and Republic of Karelian indicates a strong need to revitalize the endangered Karelian language including its all dialects. It is important to find new ways of teaching language and culture of Karelian for different age groups and language backgrounds, also provide location-independent solutions for self-studying, e.g. web based games.
The main focus in this study is Viena Karelian dialect, which is mainly spoken in North-Viena and Northern Finland. The dialect is especially needed for a communication between Finns and Viena Karelians, because they have a long history together and relatives and friends in both side of the border. It is urgent to revitalize Viena Karelian via gamification in order to support new generations to learn language and culture. Language and culture are considered as non-gaming contexts and traditionally they can be learned by using studying, reading books, and having social interactions with people who can speak the language. The concept of gamification can be utilized for developing learning games by applying game design elements [6], [7].

According to our knowledge, there are only few existing games to learn Viena Karelian dialect and culture. Uuši vuoši ${ }^{1}$ game is one example of mobile web games for learning Viena Karelian. This game is targeted for children and new learners. One recently published mobile game is Kielimestari 2 application which aim is to increase awareness of Finland's minority languages (Swedish, Northern Sami and Karelian). However, more games are needed for different age groups with various Karelian language skill levels. It is important to provide to beginner learners a possibility to learn Viena Karelian and culture in a fun and effective ways locationindependently. Therefore, a game prototype was developed to be easily accessible in desktop, tablet, and smartphone device context. The application allows learners to play and study the dialect and culture by using web browsers without a need to install the game. Preliminary user experience (UX) evaluation was conducted with 12 expert users during the international game conference of GamiFIN 2019, where also the first early version of the game prototype was presented [8].

This paper is structured as follow: related research is presented to give information about the related work of gamification, games, and web technology. Second, the design and implementation are presented to give a broad view of the game prototype. Third, user experience evaluation and findings are presented to describe the main findings. Fourth, discussion and conclusion are described to conclude the paper.

\footnotetext{
${ }^{1}$ https://uusivuosi.000webhostapp.com/

${ }^{2}$ https://apkpure.com/kielimestari/com.rsg.opikielta
} 


\section{RELATED RESEARCH}

The concept of gamification means that game design elements are utilized in non-gaming contexts to develop games [6], [7]. The aim of this approach is to get users to be more motivated and engaged towards learning objects [9], [10]. Typical examples of non-gaming contexts in educational settings are studying from books, learning in face-to-face settings, social interactions and traditions which was passed on from family to family by storytelling and habits. It is noticed a good approach to utilize gamification for learning language and culture issues, because this approach can increase the users' engagement and motivation [11], [12].

By utilizing the gamification approach, learning environments can be created to be more attractive and visually pleasurable and focusing on educational aspects. However, it is important to notice that because the aim of gamification is to increase knowledge and learning, the game elements can be simplified and the design does not need to focus on pureentertainment goals [13], [14]. These kinds of game elements are, for instance, levels, progress status, and score bars [6]. Educational PC or mobile games has been seen better solution than using only traditional teaching practices in the classroom settings [13], [15], [16]. One utility is that players are able to learn while playing the games with interesting and attractive gameplays for educational purposes. For instance, the study of an educational game for learning English indicated that players were able to increase their knowledge by improving listening, reading, and writing skills [17]. In gamification approach, there is a possibility to utilize audio and video materials for improving the effectiveness of learning, rather than by using only written texts [13]. Moreover, skills of listening, reading, writing, and speaking can be utilized when teaching language via educational games [18], [19].

Duolingo is a popular language learning game. It has over seven million users and almost five stars rating in the Google Play [20]. Currently, with this application, users can study 34 different languages for free. With the game, the user can study various themes for learning basic issues, for instance, foods, drinks, greetings, and numbers. The user can practice his or her skills of listening, reading, writing, and speaking. The difficulty of the gameplays will increase after each level, for example, after the user finishes the first level, he or she can continue to play the second level, which is more difficult than the previous one. Also, streak counts, progress status and score points are utilized in this game in order to depict the user's learning progress [21].

In this version of the game prototype, the design solutions were inspired by Duolingo game and it was used as the main reference because it has quite simple and easily approachable UI and the gameplays are more focused on the learning aspects. In addition, it was important factor that there are many active Duolingo users around the world, which indicates that the game can provide some good user experience aspects (e.g. engagement, motivating, and support learning). Moreover, especially the first author has played the game since 2013 and found it is suitable as a reference for developing the web game for learning Viena Karelian dialect and culture.

\section{Development of the Game Prototype}

There were five adapted phases from [22] (universal game heuristic) to develop this game, which are (1) context phase, (2) design phase, (3) production phase, (4) testing phase, and (5) release and support phase. This game and its basic functions (e.g. gameplays, progress bar, score) were designed according to ideas used in Duolingo game [21]. The game was developed by using Laravel PHP and Bootstrap CSS frameworks. In the learning tasks, freely available images are utilized and the audio examples were generated from Google translate pronunciation of Finnish language because we were not able to utilize Viena Karelian native speakers in the design phase. Finnish pronounciation was adapted because it is quite similar with Karelian pronunciation.

The game was implemented by using the Bootstrap CSS frontend framework in order to create the user interface (UI) looks more attractive, lightweight, and responsive [23] and adaptable for a mobile view [24]. The framework consists of HTML, CSS, JavaScript languages [24] and PC, tablet, and smartphone contexts are supported. Therefore, the Bootstrap framework was utilized in the view layer in order to design the UI more responsive and structured, having visually pleasurable layouts, and thus providing a good usability and user experience. The Laravel PHP framework was selected to use because it is widely used among software developers [25] and Laravel utilizes model-view-controller (MVC) architecture to handle complexities [26]. This architecture makes the code to be more structured and organized, increase the performance, and the modularity.

In this study, the main users of the game development is for people who are able to speak English or Finnish and their age varies from 20 to 50 years. Therefore, the game was implemented as easy to access and play location-independently without prior experience of the game or dialect. However, the long-term goal is to provide game for wider age groups with various language skill backgrounds.

The main idea in this game is that language and culture are learned by playing different topics and levels. When a user completes the level, then cultural information is shown. The more the users play and complete tasks, the more the user will learn, both language and culture. In this game version, seven themes were created and there are several examples with topics such as food and drink, clothing, furniture, seasons, body, number, days and months (Fig. 1). Each of these topics was possible to play in levels; easy, medium, and hard (Fig. 2). Moreover, the user was able to practice reading, listening, and writing skills in all levels. The ideology in this game implementation was that the knowledge of learners will increase when they complete every skill on every level (Fig. 3). The user was "rewarded" after completing each level by proving cultural information in order to teach about Viena Karelian culture, for instance, religion, architecture, traditions, events, handcrafts, and books (Fig. 4). 


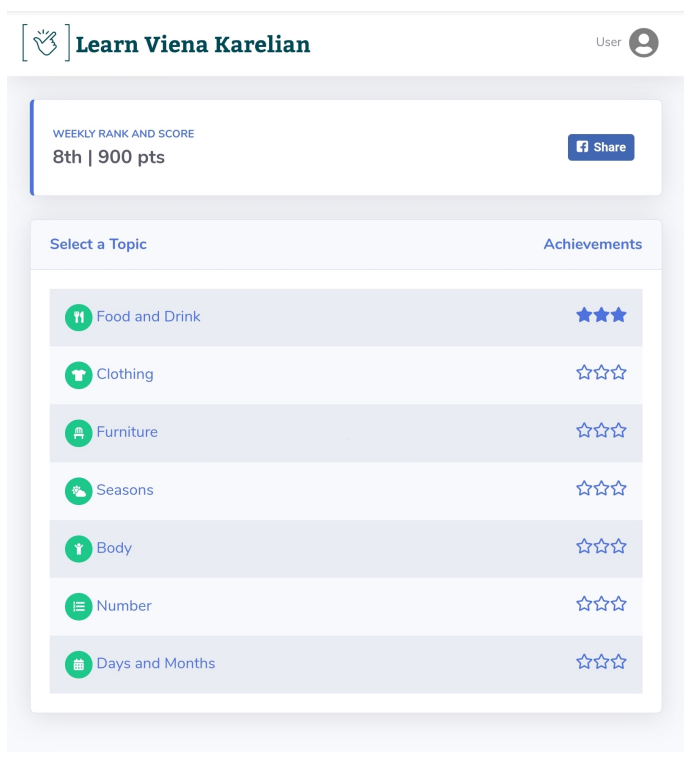

Fig. 1. Homepage: a user can select topics, check achievements and points

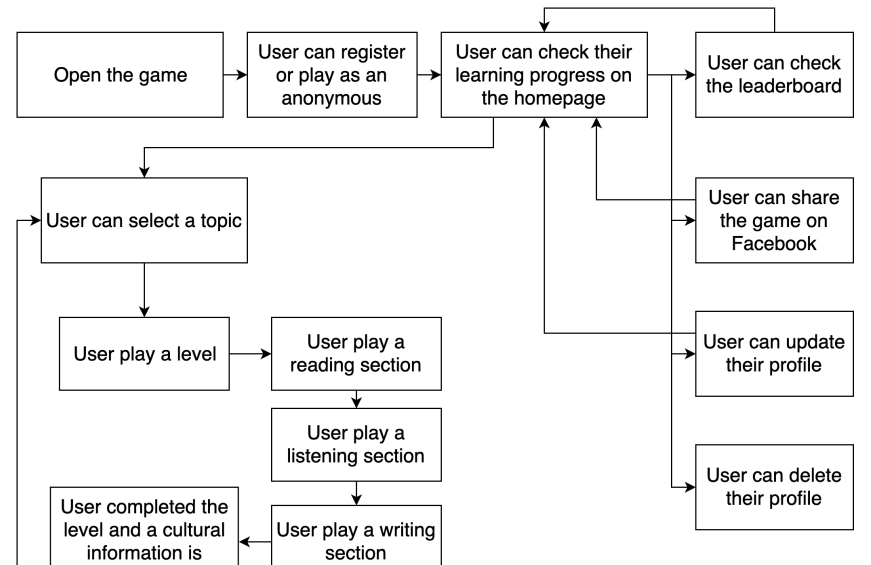

Fig. 2. Game Flow

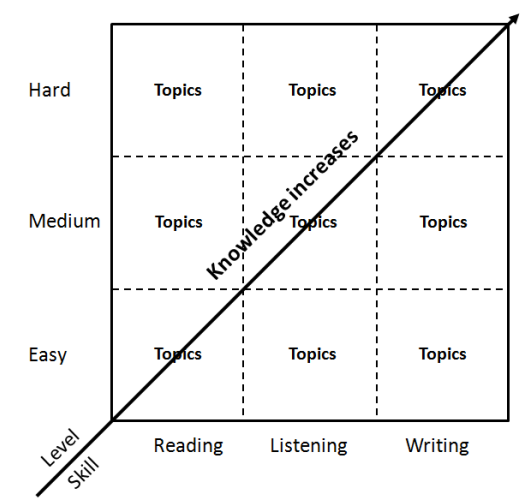

Fig. 3. Language learning model in the game prototype

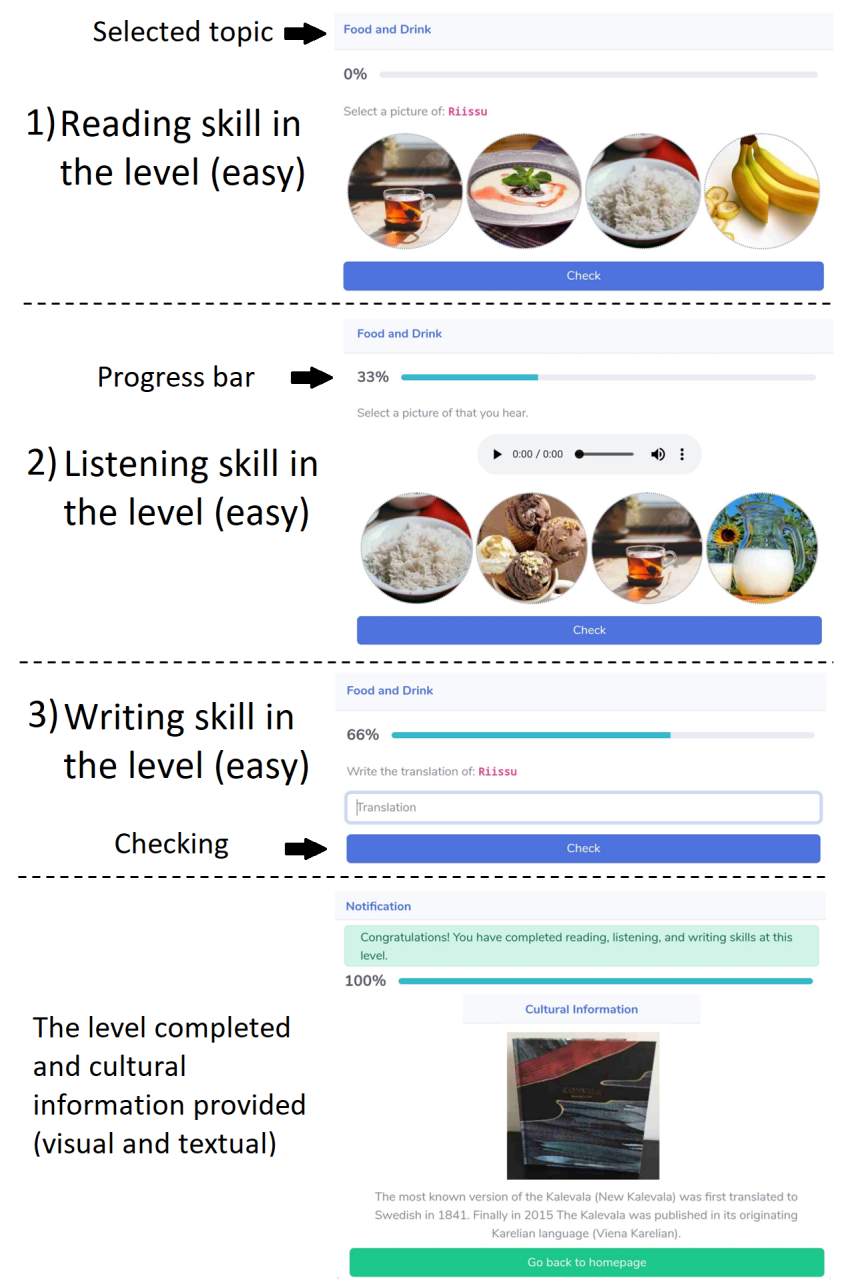

Fig. 4. An example of the gameplay with an easy level. After completion of the Reading (1), Listening (2) and Writing (3) skills in the level, the cultural information is provided in visual and textual format.

A user can learn the dialect and culture with a single player mode. Learners can play the game every day to achieve a better understanding of Viena Karelian dialect and culture, where the progressive achievement of topics can be found on the home page. In-game time is based on real-life time, so learners can see their weekly score and they can track their learning progress. The game platforms are desktop, tablet, and smartphone devices with minimum width 320px to $1281 \mathrm{px}$ (portrait). The game can be accessed with web browsers such as Google Chrome, Mozilla Firefox, and Opera.

The game elements and settings are based on Viena Karelian dialect and culture to keep the interest of learners. The content for language examples and cultural information in-game were adapted from several sources such as Viena Karelian books by Olga Karlova [27] and Pekka Zaikov [28], OmaMua newspaper ${ }^{3}$ and The Dictionary of Karelian [29]. In addition to the textual cultural information, Karelian related pictures

${ }^{3} \mathrm{http}: / /$ omamua.ru/ 
were provided as well such as pictures of mittens knitted according to Viena Karelian models or books such as Kal'evala vienankarjalakši [30] (Fig. 4). Mock-up of user interfaces were developed with a cloud service, https://balsamiq.cloud, which can be used to design wireframes with very minimum effort based on the low-fidelity design.

\section{USER EXPERIENCE EVALUATION}

\section{A. Test Settings and Participants}

User experience tests were conducted with participants of the international game conference (GamiFIN 2019). 12 game experts feedback were received with a form of questionnaire. The online questionnaires consisted of background questions and user experiences. Users were briefly asked about their prior game experiences and how they experienced the game after they had used it for a while. Users evaluated the game by making the 5-point selections for twelve adjective pairs (such as Difficult to use - Easy to use). The method was adapted from [31] and applied for the mobile serious game context. In this study, online adjective pair scale selections were used instead of asking users to select a certain amount of the given set of adjective cards like in [31].

The participants conducted the game evaluation either with or without the researcher's presence according to their own wish. It was also possible to test the game online without any assistance. Most of the participants were experts, for example, half of the users were researchers. Other occupations were UI/UX designer, game design specialist, professor and $\mathrm{PhD}$ students. It was important to collect user experiences from expert users in order to get feedback related to implementation of gamification elements. However, these participants did not have prior experiences of Viena Karelian dialect. Participants age varied from 20 to 47 years old, while average was 34 years old. 75 percentage of participants were male, and only 17 percentage of the users were native Finnish speakers. Other participants were from Brazil, Greek, Indonesia, Italy, Russia, Taiwan and Ukraine.

The first user task was to open the provided link and then either register by giving own name or nickname or select to play as an anonymous. After this, in the first page (Home) a participant's task is to select a theme to start to play (Fig. 1). When a user select a topic, a gameplay starts (Fig. 4). In this game version, each topic included three skills, which were reading, listening, and writing. All these skills were practiced in easy, medium and hard levels. The flow of gameplay was implemented as easy as possible. Therefore, when the user gets a task, he/she only needs to make a selection (reading, listening) or give answer (writing) and then tap the "Check" button. After this the notification (wrong or right answer) was shown to the user and he/she was able to continue.

\section{B. Findings}

According to the test with game expert users, the game was regarded as engaging by the 92 percentage of users. The user experiences according to adjective pair scale selections (Fig. 5) shows the game was experienced as Easy to use (4.3),

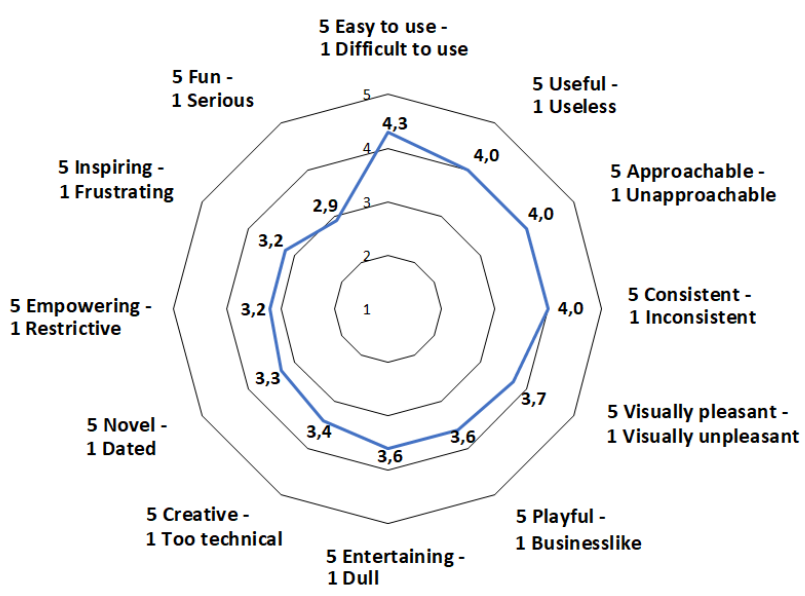

Fig. 5. User Experience Results According to Adjective Pair Scale Selections

Useful (4.0), Approachable (4.0) and Consistent (4.0). The game was regarded as more playful (3.6) than businesslike and more entertaining (3.6) than dull. However, participants perceived the game more serious game than fun (2.9).

In addition to adjective selections, valuable comments and improvement ideas were received from the participants. For instance, "Writing" exercise was experienced quite hard because when translating the given sentence a user needs to remember the exact phrase. Like one user commented: "If the translation answer is not correct, the game does not offer the possibility to learn the correct way". Also the game flow was a bit too restrictive: "It is not possible also to start the game over with learning a new word or expression in the same category unless a correct answer is provided by the user".

There were difficulties to interpret some images from each other which caused negative experiences. Also binary (right/wrong) feedback on translation tasks was regarded as too limited like one user commented: "if the right answer is "porridge" and I write "soup" (similar visual appearance) I only get "wrong answer". There should be some hints what part of the answer is correct/incorrect". In the future design, there is a need to make good image examples and also enable users to use possible synonyms. Also the game should be able to interpret misspelling like one user commented: "Understand "Sumer" as a misspelling for "Summer" - it helps non-natives of the chosen language to more easily get answers right".

In the game flow, the same word was used as an example for practicing skills (reading, listening and writing) in certain level in some topic. For instance, a user first read the Viena Karelian word "huttu" and then select one image out of four given options. When getting the correct answer, the user listen to the word "huttu" and select the correct image. User needs to translate the word "huttu" in English (porridge) or Finnish (puuro) depending on the user and selected language when starting to play the game. The goal was to provide learning experience by practicing the same word with reading, writing and listening skills. However, this solution was perceived 
negatively like one user commented: "The spoken word always seems to be the same as the previous correct shown image, there is no need to guess/learn". The other user commented the listening skill so that for a user it is easy to try to guess the example based on the previous reading skill even without a need to listen to an audio example. "In a way, it is rather easy to cheat on that" the participant said. This can mean that it is not a good solution to go to the listening skill just after reading skill with the same word or sentence example.

In future designs, there is a need to consider how to increase the level of difficulty. In Viena Karelian dialect some words are exactly the same than in Finnish language, thus some basic examples can be very easy or hard for Finnish users already in the beginning of the game for example: coffee is "kahvi" in both Finnish and Viena Karelian, but tea is "tee" in Finnish and "čäijy" in Viena Karelian. However, even though the lemma of certain words can be similar, the grammar can be totally different than in Finnish. Also, in the future developments, there is a need to use native speakers for audio examples, because for instance, the Google Finnish translation cannot pronounce "čäijy" correctly. Likewise, Karelian "š" is softer than Finnish "s". There are also other pronunciation differences, therefore, it is important to involve native Viena Karelian speakers (e.g. teachers) in the future game development projects.

\section{Discussion}

The aim of this game prototype was to provide an easy access to new learners to start to use a web game in mobile device context. The target device was tablet, but the game works well from PC and smartphone as well. It is important that the users do not need to install any application or use certain operating systems.

Our approach was to utilize simple features, which were adapted from [13]. Our language learning model in this game prototype included three levels, which were easy, medium, and hard. In addition, progress status was used for depicting the completion of levels and weekly score was shown in the home page. These are basic gamification elements, which are important to implement into game. However, in language learning game one challenge is to find balance between a level of difficulty and engagement. In the study, it seems that some tasks were too easy (e.g. reading and listening) and some too difficult (writing) in the early phase of the game play. It seems that the progress of practicing the same example (word, sentence) with reading, listening and writing skills is not maybe the best approach. Instead, the game could provide first reading and listening examples and tasks and later after a user has learned enough the writing tasks could be provided.

The game prototype included seven topics relate to basic themes such as numbers, weekdays, months, weather and eating, drinking and clothing. Cultural aspects were provided as textual and visual information relating to history, events and language learning. The aim was to provide a possibility to increase users' knowledge and learning while playing the game as presented in [13], [14]. We acknowledge that the content of language and culture examples was relatively scarce and in the future game development, there is a need to have more topics in the game, and much more cultural information should be provided. However, the aim was to show the game idea to the users and then collect their ideas and wishes for the future development.

One main challenge which relate to a development of Viena Karelian learning game, is that the dialect is very close to Finnish language, therefore some tasks are very easy and can be completed based on knowledge of Finnish language. In the future, it is important to investigate can the same game be developed for Finnish and Russian users or should the users' mother tongue be taken into account in game development.

\section{CONCLUSION}

This paper presents a game prototype, which aim was to provide an easy and pleasurable way to learn Viena Karelian dialect and culture. The preliminary user experience study was conducted with 12 expert participants. According to UX result the game prototype was experienced as motivating and engaging. According to adjective selections the game was experienced as Easy to use, Useful, Approachable and Consistent. However, the study indicated that in the future, there is a need to improve the usability of the game and provide more visual, textual and authentic audio (native speaker) content with different kind of exercises. This game prototype was developed for beginners learners. In the future it is important to develop games for different age group and people with different language background e.g. Finnish, Karelian and Russian. Likewise, it is significant to support all dialects of Karelian language (Karelian Proper (Viena Karelia (North) and South Karelian) and Olonets Karelian (Livvi-Karelian)). By providing several different games, users can help to revitalize endangered Karelian language.

\section{REFERENCES}

[1] Y. Klementyev, S. Kovaleva, and K. Zamyatin, "The karelian language in russia: An overview of a language in context," Working Papers in European Language Diversity, 12.) Helsinki: ELDIA, Tech. Rep., 2013.

[2] Rosstat, "Population size and location in russian population census 2010,” http://www.gks.ru/free_doc/new_site/perepis2010/croc/perepis_ itogi1612.htm, accessed: 2018-12-09.

[3] T. Salonen, "Karelian a digital language?" The Digital Language Diversity Project, Tech. Rep., 2017.

[4] A. Sarhimaa, "The karelian language in finland: An overview of a language in context," Working Papers in European Language Diversity, Tech. Rep., 2011.

[5] A. Sarhimaa, Vaietut ja vaiennetut, Karjalankieliset karjalaiset Suomessa. Suomalaisen Kirjallisuuden Seura, 2017.

[6] S. Deterding, "Gamification: designing for motivation," interactions, vol. 19, no. 4, pp. 14-17, 2012.

[7] S. Deterding, R. Khaled, L. E. Nacke, and D. Dixon, "Gamification: Toward a definition," in CHI 2011 gamification workshop proceedings, vol. 12. Vancouver BC, Canada, 2011.

[8] T. Damiri Burlian, P. Sharmila, P. Alavesa, and L. Arhippainen, "Revitalizing Viena Karelian Dialect and Culture with Gamification," in The 3rd International GamiFIN Conference, Gamification Group. Levi, Finland: CEUR Workshop Proceedings. April 8 10, 2019., 2019.

[9] K. M. Kapp, The gamification of learning and instruction: game-based methods and strategies for training and education. John Wiley \& Sons, 2012.

[10] B. H. Sørensen and B. Meyer, "Serious games in language learning and teaching-a theoretical perspective," in DiGRA Conference, 2007. 
[11] M. Amoia, C. Gardent, L. Perez-Beltrachini et al., "A serious game for second language acquisition," in $\operatorname{CSEDU}(1), 2011$, pp. 394-397.

[12] J. Hamari, J. Koivisto, and H. Sarsa, "Does gamification work?-a literature review of empirical studies on gamification," in 2014 47th Hawaii international conference on system sciences (HICSS). IEEE, 2014, pp. 3025-3034

[13] P. Backlund and M. Hendrix, "Educational games-are they worth the effort? a literature survey of the effectiveness of serious games," in Games and virtual worlds for serious applications (VS-GAMES), 2013 5th international conference on. IEEE, 2013, pp. 1-8.

[14] E. A. Boyle, T. Hainey, T. M. Connolly, G. Gray, J. Earp, M. Ott, T. Lim, M. Ninaus, C. Ribeiro, and J. Pereira, "An update to the systematic literature review of empirical evidence of the impacts and outcomes of computer games and serious games," Computers \& Education, vol. 94, pp. 178-192, 2016

[15] R. E. Ferdig, Handbook of Research on Effective Electronic Gaming in Education. IGI Global, 2009.

[16] H. W. Giessen, "Serious games effects: an overview," Procedia-Social and Behavioral Sciences, vol. 174, pp. 2240-2244, 2015.

[17] S. Suh, S. W. Kim, and N. J. Kim, "Effectiveness of mmorpg-based instruction in elementary english education in korea," Journal of computer assisted learning, vol. 26, no. 5, pp. 370-378, 2010.

[18] G. Stanley and K. Mawer, "Language learners \& computer games: From," TESL-EJ, vol. 11, no. 4, p. n4, 2008.

[19] P. Wouters, E. D. Van der Spek, and H. Van Oostendorp, "Current practices in serious game research: A review from a learning outcomes perspective," in Games-based learning advancements for multi-sensory human computer interfaces: techniques and effective practices. IGI Global, 2009, pp. 232-250.

[20] Google, "Duolingo in the Android OS," 2019. [Online]. Available: https://play.google.com/store/apps/details?id=com.duolingo $\{\&\} \mathrm{hl}=\mathrm{en}$

[21] "Duolingo," https://www.duolingo.com/, accessed: 2018-12-09.

[22] S. Mylly, "Genre-Based Modular Heuristic Models for Improving Usability During Game Development," Master's Thesis, University of Oulu, 2011.

[23] A. Shenoy and A. Prabhu, "Choosing Lightweight Frameworks for Intuitive Web Design," CSS Framework Alternatives, pp. 1-14, 2018.

[24] N. Jain, "Review of different responsive css front-end frameworks," Journal of Global Research in Computer Science, vol. 5, no. 11, pp. 5-10, 2015.

[25] D.-P. Pop and A. Altar, "Designing an MVC model for rapid web application development," Procedia Engineering, vol. 69, pp. 1172$1179,2014$.

[26] R. F. Olanrewaju, T. Islam, and N. Ali, "An empirical study of the evolution of php mvc framework," Advanced Computer and Communication Engineering Technology, pp. 399-410, 2015.

[27] O. Karlova, "Vienankarjalan alkeiskuršši," 2011

[28] P. Zaikov, Vienankarjalan kielioppi: lisänä harjotukšie ta lukemisto. Karjalan Sivistysseura ry, 2013

[29] "Dictionary of Karelian. Torikka, M. (1st ed.), Joki, L. (2nd eds.) Web application by Vihtari, J." http://kaino.kotus.fi/cgi-bin/kks/karjala.cgi, 2009, last update: 2019-02-22. accessed: 2019-03-22.

[30] E. Lönnrot and R. Remsujeva, "Kal'evala vienankarjalakši," Karjalan sivistysseura ry, 2015.

[31] M. Sunnari, L. Arhippainen, M. Pakanen, and S. Hickey, "Studying user experiences of autostereoscopic $3 \mathrm{~d}$ menu on touch screen mobile device," in Proceedings of the 24th Australian Computer-Human Interaction Conference. ACM, 2012, pp. 558-561. 